\title{
Immunomodulatory effect of mesenchymal stem cells may depend on secretion of IL-2 and IL-10 and inhibition of TNF- $\alpha$ in pediatric hematopoietic stem cell donors and recipients
}

\author{
KATARZYNA DRABKO ${ }^{l}$, DOROTA WINNICKA ${ }^{l}$, AGNIESZKA BOJARSKA-JUNAK ${ }^{2}$, JERZY KOWALCZYK ${ }^{l}$
}

${ }^{1}$ Department of Pediatric Hematology, Oncology and Transplantology, Medical University of Lublin

${ }^{2}$ Department of Clinical Immunology, Medical University of Lublin

\begin{abstract}
In the study we aimed to estimate the number of functional MSCs by performing colony-forming unit-fibroblast $(C F U-F)$ cultures from pediatric hematopoietic stem cell transplantation recipients and their healthy donors. Secondly we compared the concentration of cytokines interleukin $2(I L-2), I L-4$, $I L-10$ and tumor necrosis factor $\alpha(T N F-\alpha)$ in stem cell serum and in supernatant of mesenchymal stem cell cultures. We included into the study 19 consecutive pairs of donors and recipients of allogeneic stem cell transplantation from one pediatric bone marrow transplantation center. Cultures of mesenchymal stem cells were performed according to the research protocol for enumeration of human mesenchymal progenitor cells using the CFU-F assay and the expansion of human mesenchymal cells in vitro (StemCell Technologies, USA). Interleukin 10 and TNF- $\alpha$ concentration were measured by high sensitivity (Quanikine HS) human immunoassay (R\&D and Bender, USA). Interleukin 2 and IL-4 concentration were assessed by human IL-2 ELISA Version 2 assay (Bender MedSystems, Austria). The number of CFU-F colonies in cultures increased with the dose of mononuclear cells given, but the difference between donors and recipients was not significant. The TNF- $\alpha$ level significantly decreased, and IL-10 and $I L-2$ increased in CFU-F supernatant as compared to stem cell serum. The concentration of IL-4 was higher in CFU-F cultures, although p value in donors was not significant. We concluded from the study that the immunomodulatory effect of donor and recipient MSCs may partially depend on secretion of IL2 and $I L-10$ and inhibition of TNF- $\alpha$ secretion.
\end{abstract}

Key words: mesenchymal stem cell (MSCs), interleukin 2 (IL-2), interleukin 10 (IL-10), tumor necrosis factor $\alpha(T N F-\alpha)$

(Centr Eur J Immunol 2013; 38 (3): 358-362)

\section{Introduction}

Mesenchymal stem cells (MSCs) are a population of pluripotent cells occurring in the bone marrow and, under the influence of appropriate stimulation, they may differentiate towards various tissues including bone, cartilage and fat [1]. Human MSCs have immunosuppressive and anti-inflammatory properties, which are independent from human leukocyte antigens (HLA) [2]. Mesenchymal stem cells are currently used in immunosuppressive therapy of different autoimmune diseases. Although the clinical effect of MSCs is confirmed, the mechanism of their immuno- modulatory effect is not fully understood. Mesenchymal stem cells, part of the bone marrow microenvironment, are believed to play a role in proliferation, differentiation and maturation of the hematopoietic cells. In clinical settings, MSCs have been used for supporting hematopoiesis, treatment of Graft-versus-Host Disease (GvHD) [3] or repair of mesenchymal tissues [4]. Recent studies suggest that secretion of cytokines may play a key role in the immunosuppressive properties of MSCs.

Interleukin 2 (IL-2) is known to be crucial in activating T lymphocytes upon antigen stimulation and acts by differ-

Correspondence: dr hab. n. med. Katarzyna Drabko, Department of Pediatric Hematology, Oncology and Transplantology,

Medical University of Lublin, Chodźki 2, 20-093 Lublin 
ent IL-2 receptors, which are located on both naïve T-cells and memory T-cells. Stimulation of T-helper cells increased B-lymphocytes activity through one of the IL-2 receptors $[5,6]$. Most commonly used immunosuppressive regiments are based on inhibition of IL-2 receptor [7]. Tumor necrosis factor $\alpha$ (TNF- $\alpha$ ) plays a main role in development of graftversus-host disease (GvHD), one of the most serious immunologic complications after hematopoietic stem cell transplantation [8, 9]. Interleukin 10 has immunosuppressive properties and is involved in the development of anergy in specific T-cells [10] while IL-4 has a potential antiinflammatory effect and can inhibit production of TNFs [11].

\section{Aim}

In the study we aimed to estimate the number of functional MSCs by performing colony-forming unit-fibroblast (CFU-F) cultures from pediatric hematopoietic stem cell transplantation recipients and their healthy donors. Secondly we compared the concentration of cytokines IL-2, IL-4, IL-10 and TNF- $\alpha$ in bone marrow and in supernatant of mesenchymal stem cells cultures.

\section{Material and methods \\ Patients}

We included in the study 19 consecutive pairs of donors and recipients of allogeneic stem cell transplantation from one pediatric center. Patients with non-malignant disease were excluded from the study. There were 13 males and 6 females and the median age of the recipients was 4.5 years (range: 8 months-17 years) and the median age of donors was 15 years (range: $4-49$ years). The median number of transfused CD 34 cells was $2.2 \times 10^{6} / \mathrm{kg}$ body weight (range: 0.9-12). Diagnosis and clinical details of the patients are presented in Table 1.

\section{Mesenchymal stem cell cultures}

Cultures of mesenchymal stem cells were performed according to the research protocol for enumeration of human mesenchymal progenitor cells using the CFU-F assay and the expansion of human mesenchymal cells in vitro [12].

Reagents: basal medium (Human), mesenchymal stem cells stimulatory supplements (Human) and technical manual were obtained from the manufacturer (StemCell Technologies, USA). Six cultures from each sample of bone marrow or peripheral blood stem cells were performed using twice: $0.5 \times 10^{6}, 1 \times 10^{6}$ and $2 \times 10^{6}$ mononuclear cells. Colonies were counted under the microscope and average enumeration of two colonies, with identical cell dose given, was recorded.

\section{Cytokine concentration}

Bone marrow or peripheral blood stem cells serum, as well as supernatant from cell cultures were frozen in $-70^{\circ} \mathrm{C}$ before analysis. Interleukin 10 and $\mathrm{TNF}-\alpha$ concentration were measured in bone marrow serum and in supernatant from CFU-F cultures by high sensitivity (Quanikine HS) human immunoassay (R\&D and Bender, USA). The mean minimum detectable dose of IL-10 was $0.5 \mathrm{pg} / \mathrm{ml}$ (range: $0.08-50$ ) and TNF- $\alpha-0.12 \mathrm{pg} / \mathrm{ml}$ (range: 0.06-0.32) according to the test manufacturer. Interleukin 2, IL-4 concentration were assessed by human IL-2 ELISA Version 2 assay (Bender MedSystems, Austria). Limits of detection of IL-2 and IL-4 were 9.9 and $1.32 \mathrm{pg} / \mathrm{ml}$, respectively.

Statistical analysis and data presentation were prepared using computer software Statistica for Windows 7.0. The study was approved by the ethical committee of the Medical University of Lublin.

\section{Results}

The number of CFU-F colonies in cultures increased with the dose of mononuclear cells given. There was a trend to a higher number of CFU-F colonies in recipients of hematopoietic stem cell transplants as compared to their donors, but in our study this difference was not statistically significant. Results are shown in Table 2.

The median concentration of IL-2, IL-4, IL-10 and TNF- $\alpha$ did not differ between patients and healthy donors either in stem cell serum or in CFU-F cultures supernatant.

Despite the difference in the number of CFU-F colonies there was no statistically significant variation between cytokines concentration in supernatant of CFU-F cultures containing $0.5,1$ or $2 \times 10^{6}$ stem cells. The results split into three kinds of cultures are presented in Table 3.

Table 1. Clinical details of the patients

\begin{tabular}{ll}
\hline Age of the patients & \\
\hline median & 4.5 years \\
\hline min & 8 months \\
\hline max & 17 years \\
\hline Sex & 13 \\
\hline male & 6 \\
\hline female & 7 \\
\hline Diagnosis & 5 \\
\hline acute lymphoblastic leukemia & 5 \\
\hline acute myeloblastic leukemia & 1 \\
\hline myelodysplastic syndrome & 1 \\
\hline non-Hodgkin lymphoma & 1 \\
\hline chronic myeloblastic leukemia & \\
\hline Donor & \\
\hline match related & 10 \\
\hline match unrelated & \\
\hline mismatch related & 13 \\
\hline
\end{tabular}


Table 2. Median (range) number of CFU-F colonies in cultures in donors and recipients

\begin{tabular}{|c|c|c|c|c|}
\hline & \multicolumn{3}{|c|}{ Median number of CFU-F colonies } & \multirow[t]{2}{*}{$\chi^{2}$ test } \\
\hline & Dose $0.5 \times 10^{6} \mathrm{MNC}$ & Dose $1 \times 10^{6} \mathrm{MNC}$ & Dose $2 \times 10^{6} \mathrm{MNC}$ & \\
\hline Recipients & $8(1-30)$ & $15(4-36)$ & $24(3-45)$ & $p=0.39$ \\
\hline Donors & $2(1-28)$ & $5(1-18)$ & $11(3-34)$ & $p=0.048$ \\
\hline
\end{tabular}

A significant difference in the concentration of cytokines in stem cells serum and CFU-F supernatant, with respect to the cell source (donor or recipient), was found. Tumor necrosis factor $\alpha$ level significantly decreased in cultures in donors and recipients ( $p=0.001$ and 0.004 , respectively). The concentration of IL-10 was higher in CFU-F supernatant as compared to bone marrow plasma (donors $p<0.0001$ and recipients $p=0.01$ ). Interleukin 2 increased in cultures supernatant significantly in both groups, however again a greater difference was observed in donors $(p<0.0001)$ than in their recipients $(p=0.002)$. The concentration of IL-4 was higher in CFU-F cultures, although $p$ value in donors was not significant and in recipients it reached 0.01 (Table 4).

Based on these results we compared the concentration of cytokines in all tested stem cell serum specimens $(n=38)$ and all analyzed CFU-F cultures $(n=114)$. A decrease in TNF as well as an increase in IL-2, IL-4 and IL-10 in this analysis was highly significant. These data are presented in Figure 1. A statistically significant correlation was found between the number of CFU-F colonies and IL-10 concentration in cell culture supernatant from recipients (Fig. 2), however no such correlation was found in the donors $(r=0.07 ; p>0.05)$.

\section{Discussion}

Evidence of the cytokine-mediated effect of MSCs comes from the animal studies, in which the mice model of GvHD was treated with IL-10-transduced MSCs. Authors were able to demonstrate significantly lower mortality in animals treated with a higher concentration of anti-inflammatory cytokine IL-10 as compared to not transduced MSCs or controls [13]. Inhibition of allogeneic T-reactive lymphocytes response in vitro, after MSCs, was noted in anoth-

Table 3. Mean values of cytokines in supernatant of donor cultures of bone marrow and peripheral blood stem cells with respect to MNC dose in the culture

\begin{tabular}{|c|c|c|c|c|c|c|c|c|}
\hline \multirow{2}{*}{$\begin{array}{l}\text { PB/BM MNC dose } \\
\text { PB mean } n=18\end{array}$} & \multicolumn{2}{|c|}{ TNF [pg/ml] } & \multicolumn{2}{|c|}{ IL-10 [pg/ml] } & \multicolumn{2}{|c|}{ IL-4 [pg/ml] } & \multicolumn{2}{|c|}{ IL-2 [pg/ml] } \\
\hline & 0.94 & NS & 10.6 & NS & 3.34 & NS & 7.02 & NS \\
\hline $0.5 \times 10^{6} n=6$ & 0.70 & & 10.8 & & 3.21 & & 6.70 & \\
\hline $1 \times 10^{6} n=6$ & 1.04 & & 10.7 & & 3.56 & & 6.74 & \\
\hline $2 \times 10^{6} n=6$ & 1.08 & & 10.3 & & 3.24 & & 7.61 & \\
\hline BM mean $n=38$ & 0.65 & & 7.9 & & 3.53 & & 6.33 & \\
\hline $0.5 \times 10^{6} n=13$ & 0.54 & & 6.3 & & 3.67 & & 6.02 & \\
\hline $1 \times 10^{6} n=12$ & 0.59 & & 8.6 & & 3.50 & & 6.31 & \\
\hline $2 \times 10^{6} n=13$ & 0.80 & & 9.0 & & 3.41 & & 6.66 & \\
\hline Total mean $n=56$ & 0.74 & & 8.8 & & 3.47 & & 6.55 & \\
\hline
\end{tabular}

PB - peripheral blood stem cells; BM-bone marrow; $M N C$ - mononuclear cells

Table 4. Median values and standard deviation of cytokines in bone marrow serum and in CFU-F cultures in donors and recipients

\begin{tabular}{|c|c|c|c|c|c|c|}
\hline & \multicolumn{3}{|c|}{ Patients } & \multicolumn{3}{|c|}{ Donors } \\
\hline & SC serum & CFU-F supernatant & $p$ & SC serum & CFU-F supernatant & $p$ \\
\hline IL-2 [pg/ml] & $2.3( \pm 2.88)$ & $5.62( \pm 2.2)$ & 0.02 & $3.92( \pm 2.95)$ & $6.46( \pm 2.55)$ & $<0.0001$ \\
\hline IL-4 [pg/ml] & $0.54( \pm 7.73)$ & $5.22( \pm 2.73)$ & 0.01 & $1.02( \pm 7.12)$ & $2.68( \pm 2.79)$ & NS \\
\hline IL-10 [pg/ml] & $1.76( \pm 3.88)$ & $5.13( \pm 5.78)$ & 0.01 & $1.05( \pm 1.77)$ & $7.13( \pm 7.77)$ & $<0.0001$ \\
\hline $\mathrm{TNF}-\alpha[\mathrm{pg} / \mathrm{ml}]$ & $1.41( \pm 3.93)$ & $0.56( \pm 0.96)$ & 0.004 & $1.32( \pm 4.91)$ & $0.36( \pm 0.80)$ & 0.001 \\
\hline
\end{tabular}


A

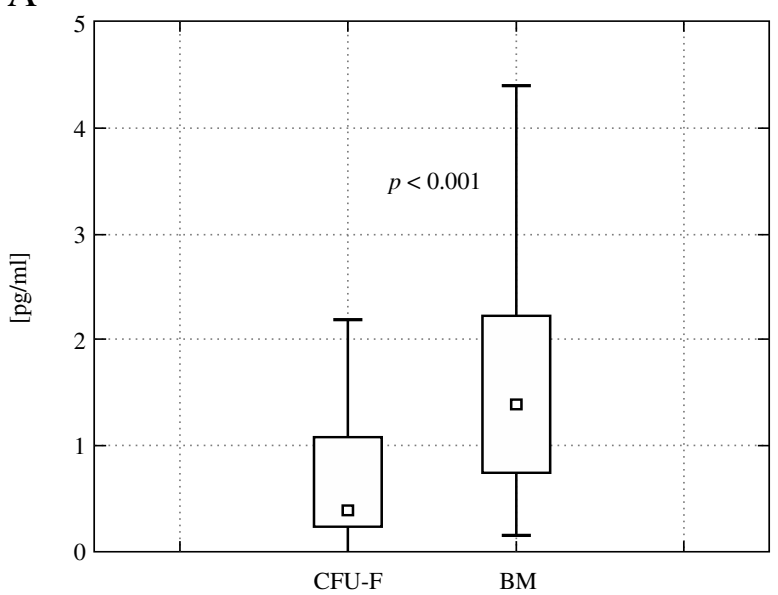

C

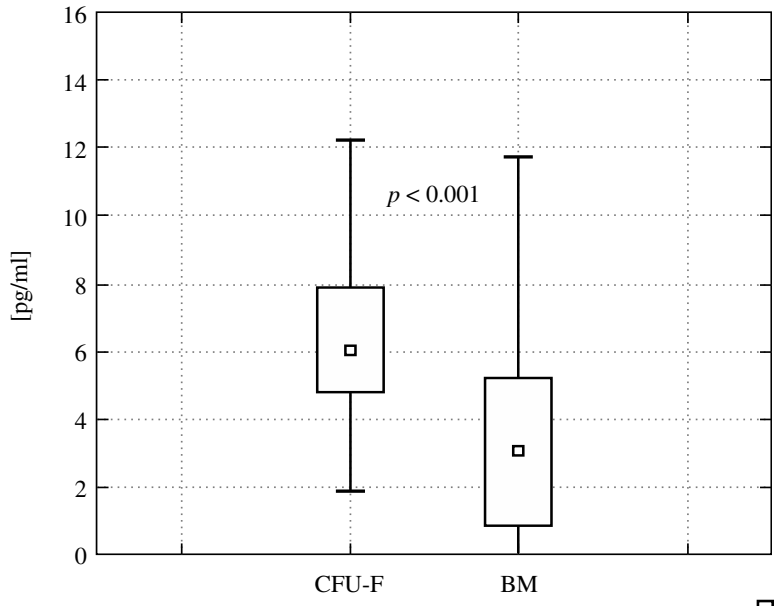

B

IL-10

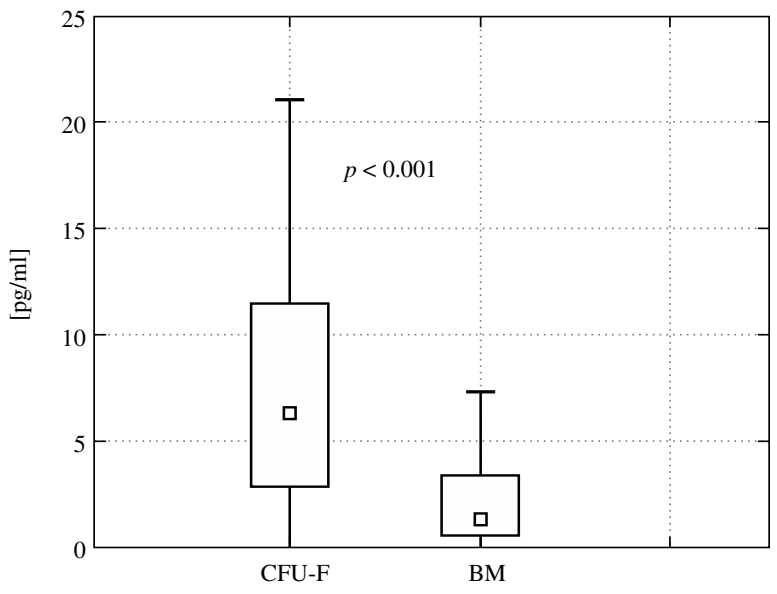

D

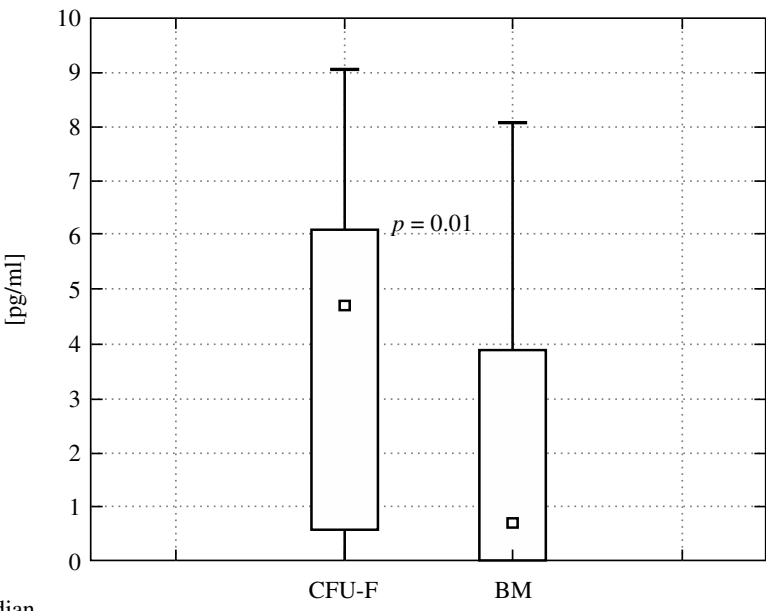

$\square$ median

$25-75 \%$

Fig. 1. Concentration of cytokines in colony-forming-unit- fibroblast (CFU-F) cultures supernatant in comparison with bone marrow (BM) serum. TNF - tumor necrosis factor $\alpha$, IL-10 - interleukin 10, IL-2 - interleukin 2, IL-4 - interleukin 4

er animal study, however the clinical effect on animals was not observed in that experiment [14]. Research performed on human MSCs, which produced divergent results observed in vitro and in vivo suggested that the mechanism of MSCs activity is complex. Immunosuppressive drugs can modify MSCs activity as it was demonstrated in cultures performed with and without cyclosporine. The result of this experiment confirmed the synergistic effect of MSCs and most popular immunosuppressant [15].

In another study, human MSCs were able to increase the level of IL-10 in supernatant of mixed lymphocyte cultures, but the level of increase was not correlated with the inhibition of lymphocyte proliferation [16]. Cytokine-mediated immunomodulatory effect of adipose tissue-derived adult MSCs comparable with bone marrow MSCs was also reported, however in this publication IL-10 concentration in cultures supernatant was below the detection level [17].

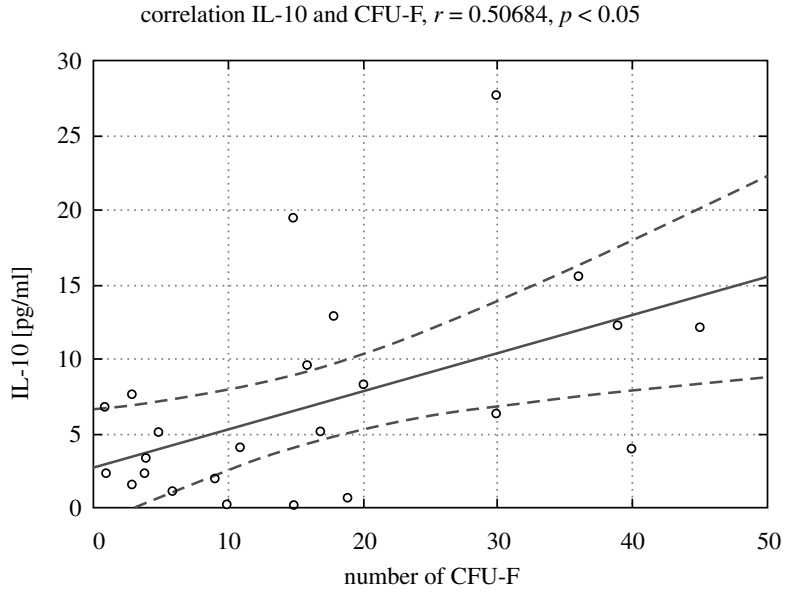

Fig. 2. Correlation was found between number of CFU-F colonies and IL-10 level in cell culture supernatant from recipients 
The role of pro-inflammatory cytokines e.g. TNF- $\alpha$ in immunological post-transplant complication is well documented and anti-TNF therapy is one of the possibilities of second-line GvHD therapy [18]. In a clinical study, anti-inflammatory cytokine IL-10 spontaneous production in unrelated bone marrow transplant recipients was reported to be associated with fever, transplant-related complications and early deaths [19]. In our patients, severe toxicities observed after stem cell transplantation did not correlate with any of the analyzed cytokines, but the study group of patients was small.

Importance of the dose of MSCs with respect to IL-2 production was described in another report. According to published data, a low number of MSCs decreases and on the contrary high MSCs doses increase IL-2 levels in cell cultures [20]. Similarly in our study, we did not observe a correlation of the number of CFU-F colonies and cytokine levels, what suggested that secretion of soluble factors is not linear.

In breast cancer, an increased level of IL-4 was considered as a part of disease pathomechanism [21], while in stem cell transplantation setting IL-4 producing CD8+ lymphocytes seemed to contribute to development of chronic Graft-versusHost Disease [22]. In our observation, a significant increase in IL-4 in cultures only from recipients suggests the role of this cytokine in the underlying disease of those patients.

In our opinion, analysis of cytokine concentration in clinical settings presented in this work may contribute to better understanding of changes in the microenvironment during the stem cell transplantation procedure and facilitate further clinical studies.

We concluded from the study that in clinical settings, the immunomodulatory effect of donor and recipient mesenchymal stem cells may be partially dependent on secretion of IL-2, IL-10 and inhibition of TNF- $\alpha$ secretion.

\section{The authors declare no conflict of interests.}

Supported by a grant of the Ministry of Science and Higher Education No. N407 11735.

\section{References}

1. Le Blanc K, Pittenger M (2005): Mesenchymal stem cells: progress toward promise. Cytotherapy $7: 36-45$.

2. Le Blanc K, Tammik L, Sundberg B, et al. (2003): Mesenchymal stem cells inhibit and stimulate mixed lymphocyte cultures and mitogenic responses independently of the major histocompatibility complex. Scand J Immunol 57: 11-20.

3. Le Blanc K, Ringdén O (2005): Immunobiology of human mesenchymal stem cells and future use in hematopoietic stem cell transplantation. Biol Blood Marrow Transplant 11: 321-334.

4. Horwitz EM, Prockop DJ, Gordon PL, et al. (2001): Clinical responses to bone marrow transplantation in children with severe osteogenesis imperfecta. Blood 97: 1227-1231.

5. Kim HP, Imbert J, Leonard WJ (2006): Both integrated and differential regulation of components of the IL-2/IL-2 receptor system. Cytokine Growth Factor Rev 17: 349-366.
6. Liao W, Lin JX, Leonard WJ (2013): Interleukin-2 at the crossroads of effector responses, tolerance, and immunotherapy. Immunity 38: 13-25.

7. Kroczek RA, Black CD, Barbet J, Shevach EM (1987): Mechanism of action of cyclosporin A in vivo. I. Cyclosporin A fails to inhibit $\mathrm{T}$ lymphocyte activation in response to alloantigens. J Immunol 139: 3597-3603.

8. Vilcek J, Lee TH (1991): Tumor necrosis factor. New insights into the molecular mechanisms of its multiple actions. J Biol Chem 1991; 266: 7313-7316.

9. Hill GR, Teshima T, Gerbitz A, et al. (1999): Differential roles of IL-1 and TNF- $\alpha$ on graft-versus-host disease and graft versus leukemia. J Clin Invest 104: 459-467.

10. Akdis CA, Blaser K (2001): Mechanisms of interleukin-10mediated immune suppression. Immunology 103: 131-136.

11. Hart PH, Jones CA, Finlay-Jones JJ (1992): Interleukin-4 suppression of monocyte tumour necrosis factor-alpha production. Dependence on protein synthesis but not on cyclic AMP production. Immunology 76: 560-565.

12. http://www.stemcell.com/ /media/Technical\%20Resources/6/ C/A/E/9/28453_mesencult_H.ashx; 2008

13. Min CK, Kim BG, Park G, et al. (2007): IL-10-transduced bone marrow mesenchymal stem cells can attenuate the severity of acute graft-versus-host disease after experimental allogeneic stem cell transplantation. Bone Marrow Transplant 39: 637-645.

14. Sudres M, Norol F, Trenado A, et al. (2006): Bone marrow mesenchymal stem cells suppress lymphocyte proliferation in vitro but fail to prevent graft-versus-host disease in mice. J Immunol 176: 7761-7767.

15. Maccario R, Moretta A, Cometa A, et al. (2005): Human mesenchymal stem cells and cyclosporin a exert a synergistic suppressive effect on in vitro activation of alloantigen-specific cytotoxic lymphocytes. Biol Blood Marrow Transplant 11: 1031-1032.

16. Rasmusson I, Ringdén O, Sundberg B, Le Blanc K (2005): Mesenchymal stem cells inhibit lymphocyte proliferation by mitogens and alloantigens by different mechanisms. Exp Cell Res 305: 33-41.

17. Puissant B, Barreau C, Bourin P, et al. (2005): Immunomodulatory effect of human adipose tissue-derived adult stem cells: comparison with bone marrow mesenchymal stem cells. $\mathrm{Br}$ J Haematol 129: 118-129.

18. Sleight BS, Chan KW, Braun TM, et al. (2007): Infliximab for GVHD therapy in children. Bone Marrow Transplant 40: 473-480.

19. Baker KS, Roncarolo MG, Peters C, et al. (1999): High spontaneous IL-10 production in unrelated bone marrow transplant recipients is associated with fewer transplant-related complications and early deaths. Bone Marrow Transplant 23: 1123-1129.

20. Fang L, Lange C, Engel M, et al. (2006): Sensitive balance of suppressing and activating effects of mesenchymal stem cells on T-cell proliferation. Transplantation 82: 1370-1373.

21. Razmkhah M, Jaberipour M, Erfani N, et al. (2011): Adipose derived stem cells (ASCs) isolated from breast cancer tissue express IL-4, IL-10 and TGF- $\beta 1$ and upregulate expression of regulatory molecules on T cells: do they protect breast cancer cells from the immune response? Cell Immunol 266: 116-122.

22. Nakamura K, Amakawa R, Takebayashi M, et al. (2005): IL-4-producing CD8(+) T cells may be an immunological hallmark of chronic GVHD. Bone Marrow Transplant 36: 639-647. 